\title{
Design and Research of Intelligent Safety Monitoring Robot for Coal Mine Shaft Construction
}

\author{
Wenjun Fu, ${ }^{1}$ Ying Xu $\left(\mathbb{D},{ }^{2}\right.$ Liangping Liu, ${ }^{1}$ and Liang Zhang ${ }^{1}$ \\ ${ }^{1}$ Beijing China Coal Mine Engineering Co., Ltd., Beijing 100013, China \\ ${ }^{2}$ Liaoning Technical University, Fuxin 123000, China \\ Correspondence should be addressed to Ying Xu; xuying@lntu.edu.cn
}

Received 15 April 2021; Revised 18 May 2021; Accepted 7 June 2021; Published 23 June 2021

Academic Editor: Sang-Bing Tsai

Copyright (c) 2021 Wenjun Fu et al. This is an open access article distributed under the Creative Commons Attribution License, which permits unrestricted use, distribution, and reproduction in any medium, provided the original work is properly cited.

This study analyzes the main causes of accidents in the period of coal mine shaft construction and the shortage of the existing safety monitoring technology, puts forward the intelligent safety monitoring robot technology based on shaft construction, and deeply investigates the functions that the safety monitoring robot should have. Besides, the research objective of intelligent safety monitoring robot for shaft construction is pointed out, and the research is carried out from such aspects as the robot body structure, walking mechanism, adsorption mechanism, control, communication, intelligent sensing, hazard source recognition, recognition of explosive detonators, and software platform development. As well, this study designs the technical device of intelligent safety monitoring robot for shaft construction, defines the characteristics of this technology, expounds the important significance of studying this technology, and indicates the development trend of this technology.

\section{Introduction}

With the increasing social demand for coal and constant improvement of mining capacity, the increasing depth of mining is an inevitable trend of mining [1]. The coal resources in China are predicted to be 1844.048 billion tons in the vertical depth of $1000 \mathrm{~m}$, among which the reliable coal resources are 916.910 billion tons, accounting for $40.5 \%$ of the predicted total amount. There are 1340.375 billion tons of coal resources in the buried depth of 1000-1500 m, among which the reliable coal resources are 667.691 billion tons, accounting for $29.4 \%$ of the predicted total amount. There are 1367.681 billion tons of coal resources in the buried depth of 1500-2000 m, among which the reliable coal resources are 329.229 billion tons, accounting for $30.1 \%$ of the predicted total amount. With the exploitation of deep coal resources, the depth of coal mine shafts [2] is keeping breaking records, and higher requirements are put forward for coal mine construction, especially deep shaft construction [3]. Due to the coupling action of temperature and pressure on deep rocks, coupled with the rock characteristics of strata, complex stratification, and joint structure, the shaft construction is faced with a variety of disasters such as water damage, gas, lifting, transportation, and falling $[4,5]$. If the borehole blasting method is used in the construction process [6], a large number of dust particles and harmful gases such as carbon dioxide will be produced. If they are not eliminated in time, they will seriously harm the physical and mental health of the construction workers. Meanwhile, when the concentrations of dust and methane reach a certain level, it is easy to cause explosion, fire, and other accidents, threatening the life safety of construction workers $[7,8]$. In addition, due to the small space of the shaft, coupled with serious dust and fog, small visibility, and large quantities of cables and equipment, there are many potential safety hazards. If the inspection is not timely enough, it will seriously endanger the life safety of the workers $[9,10]$.

Accidents often happen suddenly and unpredictably during shaft construction and usually cause serious consequences [11]. Figure 1 shows the accident statistics of an enterprise.

With the increase in the depth of shaft construction, the difficulty of shaft safety inspection is also increasing. Seeing from information science, risks derive from the backward safety monitoring means $[12,13]$, the nontimely collection of underground dangerous rock environment information, 


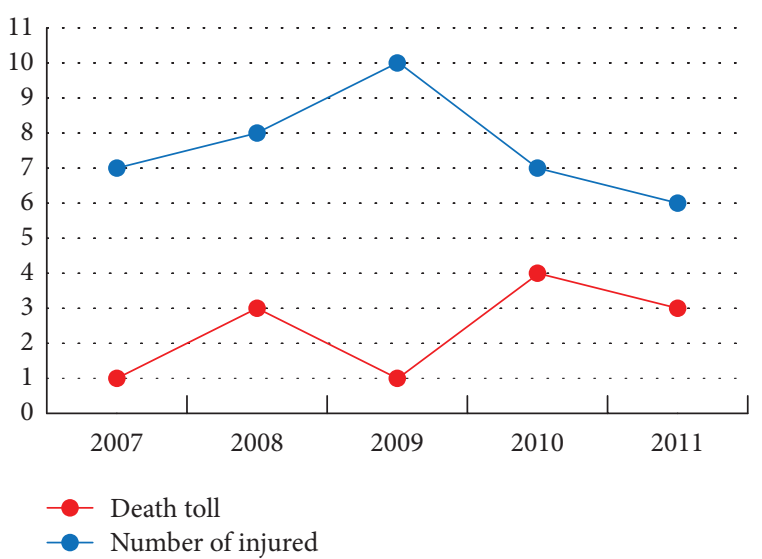

FIgURE 1: Roof falling accident of a group enterprise from 2007 to 2011.

the imperfect information analysis and processing, and the insufficient information sharing and utilization. Thus, intelligent safety monitoring of shaft construction is a problem to be solved urgently $[14,15]$. Due to the closed and dispersed safety information of new shafts, it is difficult to obtain complete and detailed real-time information for more effective monitoring and intervention in advance in the construction and production of mines [16, 17]. Hence, in order to realize the safety and efficiency of shaft construction, the key is to improve the intelligent and unmanned shaft safety monitoring and information collection [18, 19]. Therefore, it is urgent to study the intelligent safety monitoring robot technology and equipment of shaft construction to replace current manual safety monitoring and inspection.

In recent years, the intelligent inspection robot for mine construction, which combines coal mine safety monitoring and robot technology, has been widely used in the field of underground safety inspection [20]. The inspection robot can carry out long-time safety inspection according to the scheduled inspection tasks [21], collect a lot of real and accurate monitoring data in the process of inspection, and immediately transmit the data to the background data platform via the private underground wireless network $[22,23]$. Meanwhile, the comprehensive analysis and calculation of multiple data are conducted according to the software algorithm designed in advance, and the threshold values are compared for a sound-light alarm [24, 25]. Based on the advancement and intelligence of the intelligent inspection robot for mine construction, studying the robot automation system more suitable for underground safety inspection conforms to the development requirements of intelligent mine.

The wall-climbing robot can walk on the vertical facade. Because it can move freely on the wall with fewer barriers, the wall-climbing robot has received more and more attention in the engineering field [26]. The wall-climbing robot can enable people to get rid of dangerous jobs and easily complete high-altitude operations that are difficult for human beings to complete, so it is widely applied in building surface cleaning, tank crack inspection, fire rescue, ship rust removal, and other fields [27-33]. The key technologies of the wall-climbing robot mainly focus on the adsorption ability and movement ability [34]. How to solve the obstruction effect of its gravity on wall-climbing movement? $[35,36]$. Researchers around the world have tried all sorts of things, such as magnetic adsorption, negative pressure adsorption, electrostatic adsorption, and climbing adsorption. Each technology has its advantages and disadvantages and should be selected according to the working environment and specific needs. Similar to the ground-walking robot, the wall-climbing robot mostly moves with foot-type, wheeltype, crawler-type, and mixed mobile platforms [37, 38]. It just moves under the adsorption capacity that counteracts gravity, and there are higher requirements for the motion system control design [39-44].

This study innovatively applies the robot system available for shaft wall walking and monitoring to the safety inspection of coal mine shaft construction by adopting negative pressure adsorption technology. Through process control and algorithm design, the system can realize the robot adsorption and the dynamic balance of power requirements for walking, the fusion calculation of multidimensional and multisensor data, and the comprehensive judgment of the overall safety level. The hazard source identification system and the incomplete explosives identification system can substitute manual detection in dangerous locations where personnel can hardly reach. A robot whole-process function design is performed, which is verified to be feasible by experiments. In the harsh and complex environment during the shaft construction period, the wallclimbing robot can replace manpower to implement intelligent inspection and improve the inspection efficiency. In case of hazardous events, it can replace manpower for inspection to reduce the probability of casualties.

\section{Analysis on Safety Monitoring Function of Intelligent Safety Monitoring Robot for Shaft Construction}

The working conditions of shaft excavation, especially blasting, are very bad. According to the particularity and safety requirements of shaft blasting operation, the safety monitoring robot should have the following safety monitoring functions:

(1) Ventilation and gas: safety regulations require that local ventilators shall not be stopped without authorization. In case of a sudden stop, personnel shall withdraw to a safe place in front of the local ventilator in time. Then, the electrician, the inspector, and the local ventilator worker shall be on the scene at the same time for manual power transmission. After confirming safety, personnel can enter the workplace. According to this requirement, the safety monitoring robot must have the function of gas and wind speed monitoring.

(2) Comprehensive dust prevention: the working face roadway must be washed regularly and should be kept wet so that dust does not fly when walking. The 
safety monitoring robot should have the function of dust monitoring.

(3) Shaft wall management: in case of high pressure of the shaft wall, shaft wall separation, supporting plate deformation, an increase in net packs, and sound from the shaft wall, it is required to stop working immediately and evacuate all personnel from the working face. After the shaft wall becomes stable and the reinforcement is made from the outside to the inside, the operation can continue. The safety monitoring robot should have the function of shaft wall deformation monitoring.

(4) Blasting management: during blasting, a warning range must be set, and no one is allowed in the warning range. The hidden dangers discovered must be treated before others can be allowed to work. Thus, the safety monitoring robot should also have the function of dust, carbon monoxide detection, carbon dioxide detection, miss-fire explosive recognition, and sound and light warning.

(5) Water control: in the event of the increase in water from the showerhead, red on the wall, water on the wall, air cooling, and fog on the shaft, it is required to immediately stop working, withdraw the personnel, and report to the superior department for orders. The robot should have the function of shaft wall seepage recognition and monitoring.

(6) Electromechanical management: the "power failure and supply" system shall be strictly implemented. According to this requirement, the robot should have the function of information integration with the power supply system to realize the auxiliary power off and transmission management.

(7) The robot software platform should have the function of system integration. The software platform can have the data and information integration function of lifting system, power supply system, ventilation system, and monitoring system, which is convenient for analysis and comprehensive evaluation so as to realize effective and full coverage of safety monitoring.

In conclusion, the safety monitoring robot should have the function of environmental dust monitoring, toxic gas monitoring, safety patrol inspection, hazard source recognition, integration and linkage with other system information, forecast, and alarm in the coal mine shaft construction process.

\section{Research Objective of Intelligent Safety Monitoring Robot for Shaft Construction}

In current robot studies, the wall-climbing robot technology is changing quickly, and its application fields are gradually expanding. For example, the wall-climbing robot is used to clean the glass outside the building, maintain containers, and check safety. In some fields, the wall-climbing robot has been able to do some heavy and dangerous work instead of humans. This study aims to study the intelligent wallclimbing shaft safety monitoring robot. The robot can crawl on the surface of the shaft wall and is responsible for safety monitoring and inspection during the construction period of the shaft. The robot has a modular design and can be adsorbed on the surface of the shaft wall, with a special walking mechanism. The robot can crawl and patrol independently on the shaft wall, realize the shaft survey under the remote control of beyond human visual distance, and have the functions of local autonomous navigation and electronic map construction. At ordinary times, the robot can inspect the safety state of the shaft wall, the airflow velocity in the shaft, and the dust content in the shaft. The intelligent sensor can monitor methane, carbon monoxide, and other toxic and harmful gases and wirelessly transmit monitoring data and construction site images to the ground. The robot is designed with a sound and light alarm device. Based on intelligent video and multisensor information integration technology, the robot can intelligently identify miss-fire, shaft wall deformation, shaft wall water seepage, and other dangerous sources and give timely warning. The robot provides the site basis for operators to judge the site situation and make the decision of the operation task, which can effectively guarantee the safety of operators and improve the efficiency of shaft construction.

\section{Design of Intelligent Safety Monitoring Robot for Shaft Construction}

Based on the environmental characteristics of shaft construction and the needs of shaft construction safety inspection, it is necessary to conduct in-depth research and design of the robot body structure, walking mechanism, adsorption mechanism, control mechanism, intelligent sensing components, communication mode, and software platform.

4.1. Robot Body Structure. There are more than ten kinds of harmful gases in coal mines, including methane, carbon dioxide, carbon monoxide, hydrogen sulfide, sulfur dioxide, nitrogen dioxide, ammonia gas, hydrogen gas, radon gas, and nitrogen gas. The shaft is a channel between the coal mine and the outside. Due to the particularity of the coal mine shaft environment, there is a combustible explosive gas, and the electrical equipment in the shaft shall meet the safety design requirements of mining products and meet relevant provisions of the national standard GB3836. The load of the wall-climbing robot directly affects its reliable adsorption on the shaft wall, and the large load requires a large adsorption force. Meanwhile, the design difficulty of the adsorption device also increases. Hence, the key to robot design is an effective reduction of the weight of the robot. In order to adapt to the space of the coal mine shaft, the robot structure should be designed as compact as possible. In order to meet the requirements of explosion suppression and reduce the weight of the robot, the intelligent safety monitoring robot for coal mine shaft construction adopts the design of explosion suppression and intrinsic safety. The 
shell is made of stainless steel, which not only is corrosionresistant but also can increase the strength of the robot and improve its impact resistance capability. The internal structure components are made of light materials such as carbon fiber, which can effectively reduce the weight of the robot. The robot body is mainly composed of actuating shaft, driving wheel, ball bearing, driving motor, transmitting antenna, communication device, image acquisition device, tripod head, control device, intelligent sensing device, negative pressure adsorption device, and shell, as shown in Figure 2.

The bottom structure of the robot is shown in Figure 3, and it is composed of a driving wheel, driving motor, adsorption disc, and mechanisms related to robot walking.

4.2. Design of Walking Mechanism of the Robot. The safety monitoring robot needs to crawl and move freely on the surface of the shaft, so it is necessary to study and design the walking mechanism of the robot so as to achieve flexible control. According to the driving theory principle of the automobile, the intelligent safety monitoring robot for shaft construction has the four-wheel driving mode, and the four wheels are separately and differentially driven by the motor. The robot is driven by four DC servo systems to provide the required torque and torque. Such structure has the following advantages: firstly, the control mode is easy to achieve. As long as the speed of the driving motor is controlled respectively, the walking path of the robot can be controlled. Secondly, zero-radius turning can be achieved, and the flexibility of the robot in the process of curve walking is improved. Besides, the carrying capacity and the running speed of the robot can be increased. Due to the limitations of working conditions on the overall structure size of the robot, the reducer is integrated into the motor, and the high-speed output shaft of the motor is connected to the input shaft of the reducer. Besides, the output shaft of the reducer is connected to the driving wheel. As shown in Figure 4, the walking mechanism consists of the motor, reducer, and walking wheel. The fitting and connection are designed reasonably, and the omnidirectional movement adaptability of the walking mechanism on the rough shaft surface is studied so that it can move flexibly while bearing the weight and load of the robot. The walking wheels are made of cast steel. To improve the adhesion property, a rubber ring is designed on the surface of the traveling cast steel wheel. To meet the special requirements of the explosion environment in the coal mine shaft, and prevent static electricity generated by friction between the rubber ring and the shaft surface, the rubber ring is made of flame-retardant and antistatic material to prevent spark caused by friction and static electricity from detonating combustible and explosive gases in the shaft so that the robot can work reliably in the explosive gas environment of coal mine shafts.

4.3. Adsorption Mechanism of the Robot. The wall-climbing robot is a special purpose robot, and the most significant characteristic that distinguishes the robot from other robots is that the robot can overcome the earth gravity and has the

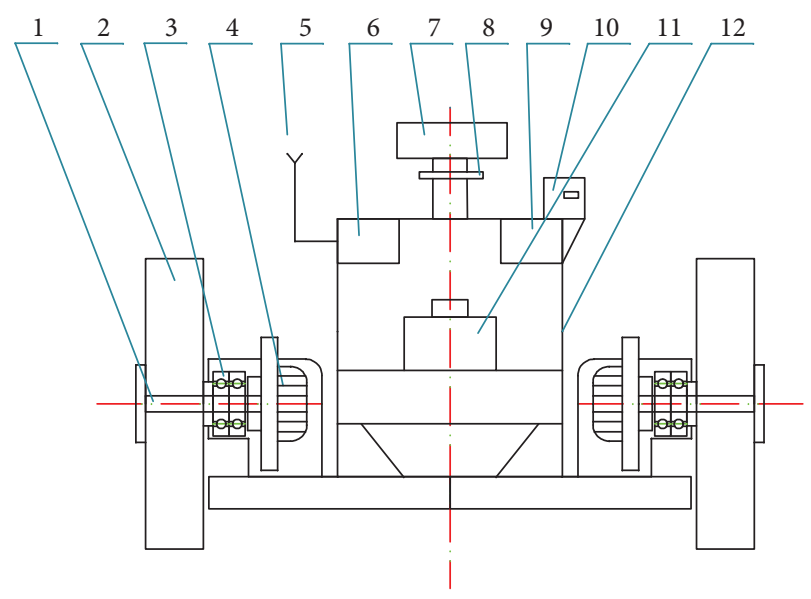

FIGURE 2: Structure diagram of intelligent safety monitoring robot for coal mine shaft construction. (1) Actuating shaft; (2) driving wheel; (3) ball bearing; (4) driving motor; (5) transmitting antenna; (6) communication device; (7) image acquisition device; (8) tripod head; (9) control device; (10) intelligent sensing device; (11) negative pressure adsorption device; (12) shell.

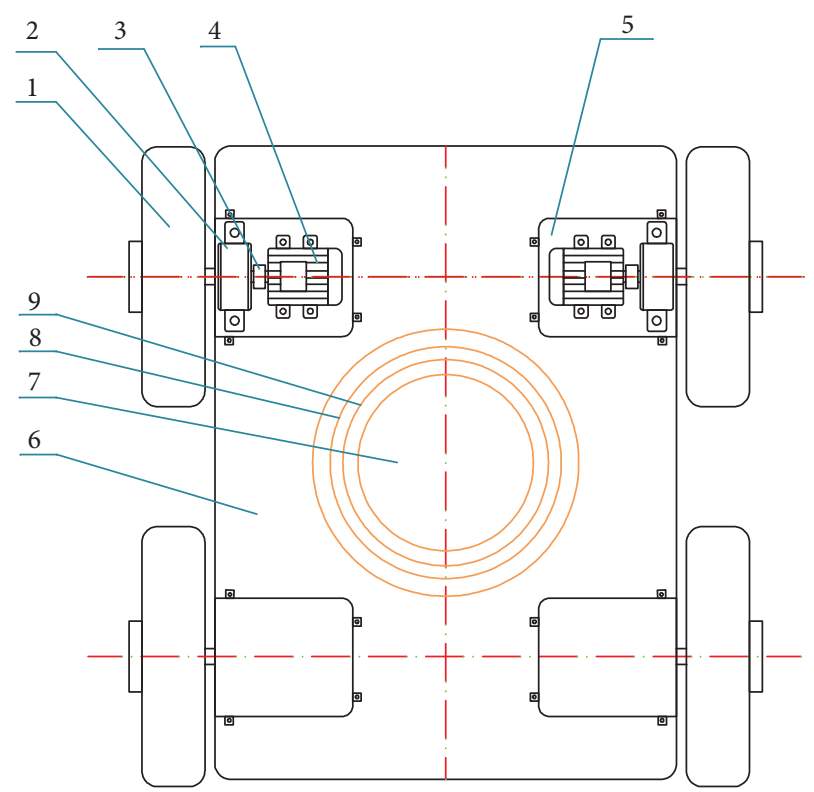

FIGURE 3: Bottom structure diagram of intelligent safety monitoring robot for coal mine shaft construction. (1) Driving wheel; (2) bearing pedestal; (3) actuating shaft; (4) driving motor; (5) motor base; (6) shell; (7) adsorption disc; (8) gasket 1; (9) gasket 2.

ability to remain stationary and move flexibly on the inclined, vertical, or inverted wall surface. At present, the adsorption methods of the wall-climbing robot mainly include magnetic adsorption, negative pressure adsorption, and propeller push. In recent years, gel adsorption, dry adsorption in imitation of gecko feet, wet adsorption in imitation of snail, grasping like rock climbing, and burr grasping have appeared. Magnetic adsorption includes permanent magnet adsorption and electromagnetic adsorption. For such adsorption methods, the robot is only 


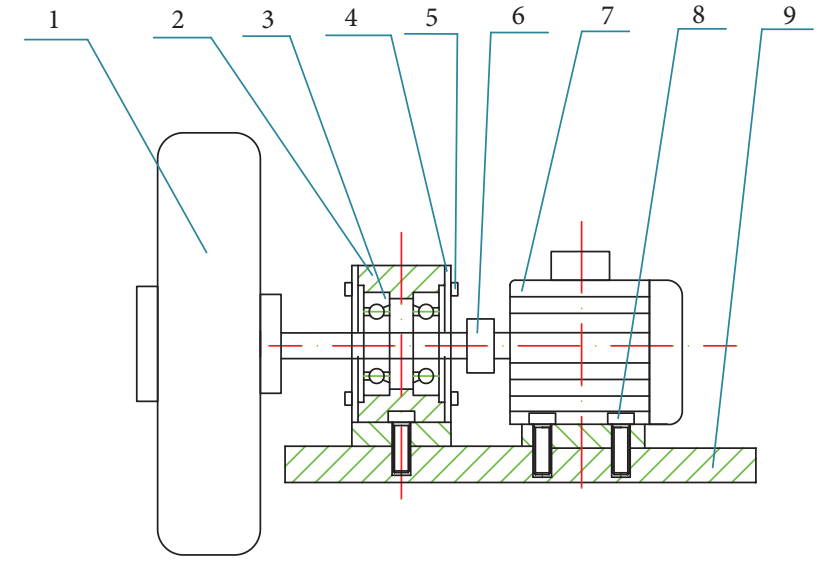

FIGURE 4: Walking mechanism of intelligent safety monitoring robot for coal mine shaft construction. (1) Driving wheel; (2) bearing pedestal; (3) ball bearing; (4) cover plate of bearing pedestal; (5) set screw; (6) connector; (7) driving motor; (8) set bolt; (9) base.

suitable for adsorption on the surface of magnetic materials such as steel and iron. The low vacuum negative pressure adsorption is to draw out the air in the negative pressure chamber of the adsorption device by the rotation of the fan to generate negative pressure for adsorption. Although the wall surface has strong adaptability, it also has the defects of high noise and large size. In the high vacuum negative pressure adsorption, a vacuum pump is used to form a certain vacuum degree in the vacuum sucker. Its advantages are low noise and small size, but there are high requirements for wall surface smoothness and air permeability. The principle of propeller push is to utilize the inward thrust generated by the propeller rotation to attach the robot on the wall through reasonably designing the angle of the propeller, but there are also the disadvantages of high noise and restricted application scenarios. The negative pressure adsorption robot has a wide range of application, free from the restriction of adsorption wall surface materials, and the low negative pressure and vacuum adsorption method has the advantages of strong wall adaptability and flexible movement, so the wall-climbing robot with low negative pressure and vacuum adsorption has been widely used.

At present, low vacuum adsorption technology is applied in a narrow range of industrial robots. It requires a high degree of working surface smoothness. The research on the sealing and friction of the adsorption mechanism cannot meet the requirements in complicated working environments. With the deficiencies in the dynamic control and electric energy distribution of walking mechanism and suction adjustment, it is designed with a single function and is able to fulfill relatively simple tasks. It also lacks powerful fusion computing power for multisensor data transmission. These shortcomings have limited the application of low vacuum adsorption technology in industrial robots.

Because the coal mine shaft is deep and the surface of the shaft wall is rough, the working safety of the robot is of great importance. The robot should overcome gravity and be able to attach safely to the surface of the shaft wall. The intelligent safety monitoring robot can be stationary and move flexibly on the wall surface with a certain incline. The bearing capacity of the adsorption mechanism is the primary problem to be considered, and the study of the negative pressure adsorption mechanism is the key to the wall-climbing robot. By analyzing the deficiencies of existing low vacuum adsorption technology, the reliability technologies of low vacuum adsorption system, such as negative pressure generation technology, automatic negative pressure holding technology, and command handshake technology, are studied. By studying the principle of new sealing technology, a sealing mechanism with a good sealing effect and small friction resistance is designed to ensure that the robot can reliably adsorb on the surface of the shaft and improve the mobility and flexibility of the robot.

The stress of the safety monitoring robot is analyzed. When the safety monitoring robot works on the shaft wall, the safety force of any posture on the inner wall of the shaft is analyzed. For simplification, the shaft wall surface is simplified as a plane, and only the stress state of the safety monitoring robot with strict upward linear motion is analyzed. When the wall-climbing robot moves upward in a straight line with angle $\theta$ at the vertical direction on the vertical plane, the force is shown in Figure 5.

According to D'Alembert's virtual work principle and force equilibrium relationship, the force equation of the linear motion of the safety monitoring robot with any posture on the vertical wall is as follows:

$$
\left\{\begin{array}{l}
N_{1}+N_{2}+N_{3}+N_{4}-F_{p}=0, \\
F_{m}-F_{f}-M_{a}-G \cos \theta=0, \\
\left(N_{1}+N_{2}\right) \cdot 2 L+G \cos \theta \cdot H-F_{p} \cdot L=0, \\
\left(N_{1}+N_{3}\right) \cdot 2 L+G \sin \theta \cdot H-F_{p} \cdot B=0,
\end{array}\right.
$$

where $M$ is robot mass; $G$ is the force of gravity on the robot; $F_{m}$ is the friction of driving wheel of the wall-climbing robot; $F_{f}$ is the friction of the wall on the sealing skirt; $N_{i}$ is the pressure of the wall acting on each walking wheel; $F_{p}$ is the equivalent resultant force on the robot body due to adsorption caused by internal and external pressure difference; $L$ is the distance from robot center of gravity to the front and rear wheels; $B$ is space between the two front (rear) wheels, $L>B$; $H$ is the distance between the center of gravity of the robot and the wall; $a$ is the accelerated speed of the robot; and $u_{1}$ is the coefficient of friction between the wheel and the wall, and the friction of the moving wheel provides the driving force for the robot, $F_{f}=u_{1} F_{p l}$.

Friction coefficients are usually measured by the angle of friction: set one of two objects as a slope and let the other object slide down the slope while gradually reducing the angle of dip angle $\theta$ until the object can slide down at a constant speed. Next, $\mu=\tan \theta$ can be obtained according to the formula $m g \sin \theta=\mu m g \cos \theta$.

If the negative pressure adsorption force generated on the sealing skirt is set as $1 / 4$ of the negative pressure adsorption force of the robot body, the minimum negative pressure adsorption force on the robot body that meets the robot motion condition is 


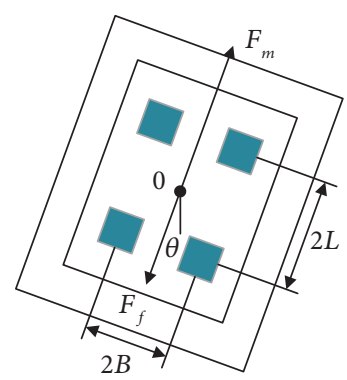

(a)

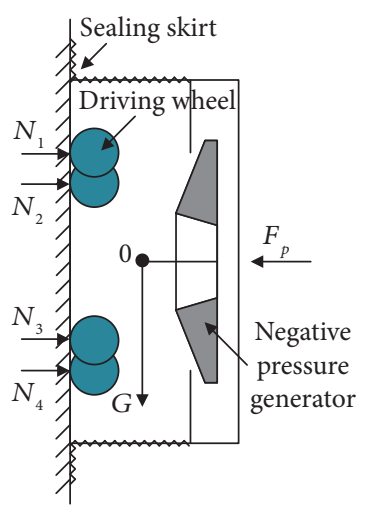

(b)

FIGURE 5: Force diagram of the wall-climbing robot with any posture.

$$
F_{p}=\frac{4 G(\cos \theta+a)}{4 u_{1}-u_{2}}
$$

where $u_{2}$ is the friction coefficient between the sealing skirt and the wall.

$$
N_{i}=\min \left(N_{1}, N_{2}, N_{3}, N_{4}\right) .
$$

When the robot is moving, 4 wheels are required to contact with the wall; that is, the condition $N_{i}>0$ must be met. Then, the minimum negative pressure adsorption force is at least

$$
F_{p}=\frac{G H(\sin \theta+\cos \theta)}{B} .
$$

Thus, the negative pressure adsorption force acting on the robot body must meet the requirements of the above two formulas, namely,

$$
F_{p}>\max \left(F_{p}=\frac{4 G(\cos \theta+a)}{4 u_{1}-u_{2}}, F_{p}=\frac{G H(\sin \theta+\cos \theta)}{B}\right) .
$$

The negative pressure of the adsorption system of the wall-climbing robot is required to be at least

$$
\Delta_{p}=\frac{F_{p}}{S_{a}},
$$

where $S_{a}$ is the effective adsorption area of the robot.

The minimum safe working negative pressure curve of the wall-climbing robot in the state of motion is shown in Figure 6 . The area represented by the vertical line was the safe adsorption and negative pressure working area of the robot, and the lower bound of the area represented the minimum negative pressure curve satisfying the safety of the robot. It can be seen that when the robot moved, the negative pressure adsorption condition was related to the attitude angle of robot, and the expression is

$$
\left\{\begin{array}{l}
\Delta p=11204.38(\cos \theta+0.2), \quad 0<\theta<0.44 \\
\Delta p=11130,0.44<\theta<1.57
\end{array}\right.
$$

Compared with the glass curtain wall and outer wall of buildings, the curvature of the shaft wall surface is small and the roughness is large, so it is very difficult to design the negative pressure adsorption equipment. Hence, the wallclimbing robot with low negative pressure and vacuum adsorption is designed. Its principle is that the rotary fan is used to draw out the air in the negative pressure chamber to generate negative pressure for wall adsorption. The negative pressure adsorption used in the design is achieved as follows: the radial-flow centrifugal fan which rotates at a high speed $(25,000 \mathrm{r} / \mathrm{min}$ above) is used to draw out the air in the adsorption chamber of the wall-climbing robot to form negative pressure. Compared with the vacuum pump, the flow rate of the fan is much greater at the same power. Although the flow of the tube-axial fan is larger than that of the radial-flow fan, the vacuum of negative pressure cannot meet the requirements of the wall-climbing robot. When the fan works normally, air flows in through the sealing skirt where the robot contacts with the shaft wall and discharges from the fan. The maximum vacuum of negative pressure is about $45 \mathrm{kPa}$, and the common working negative pressure is $10-30 \mathrm{kPa}$. Some small wall-climbing robots can work under the negative pressure of about $2-5 \mathrm{kPa}$. Through the research of robot noise reduction technology, the noise can be reduced to below $80 \mathrm{~dB}$. The robot can crawl over the rough surface of the shaft wall (the inclination of the reverse angle shall not be less than $-10^{\circ}$, and the minimum operating gap shall not be lower than $20 \mathrm{~mm}$ ). The negative pressure adsorption mechanism is composed of a sealing ring, adsorption disc, air outlet, set bolt, adsorption motor, impeller, adsorption mechanism shell sensor, and camera. The negative pressure adsorption mechanism of an intelligent safety monitoring robot for shaft construction is shown in Figure 7.

Under different inlet conditions for the centrifugal fan, the comparison curve between the input voltage of the driving motor of the centrifugal fan, the output power, and output negative pressure of the negative pressure generator is shown in Figure 8, where the red curve indicates the test data when the air inlet was fully opened, while the blue curve indicates the test data when the air inlet was fully closed. 


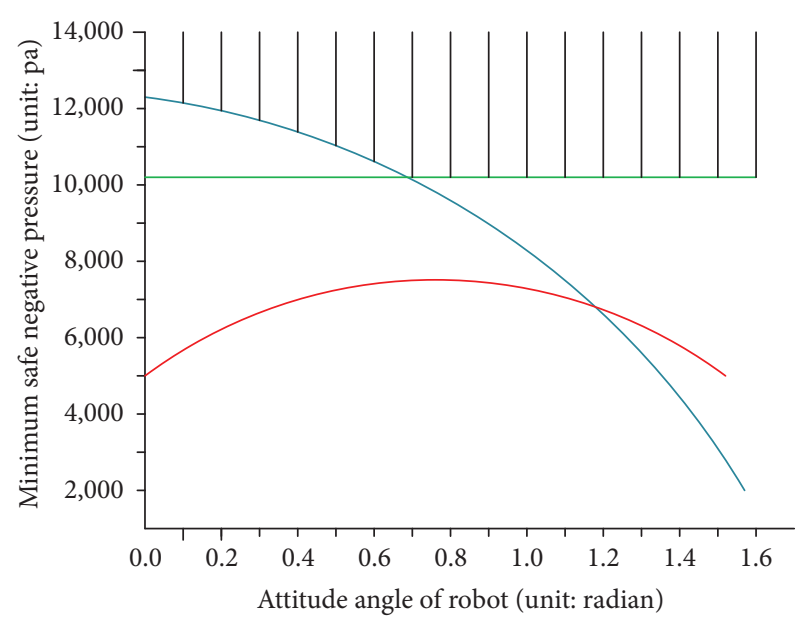

Figure 6: Suction pressure needed while the robot is moving.

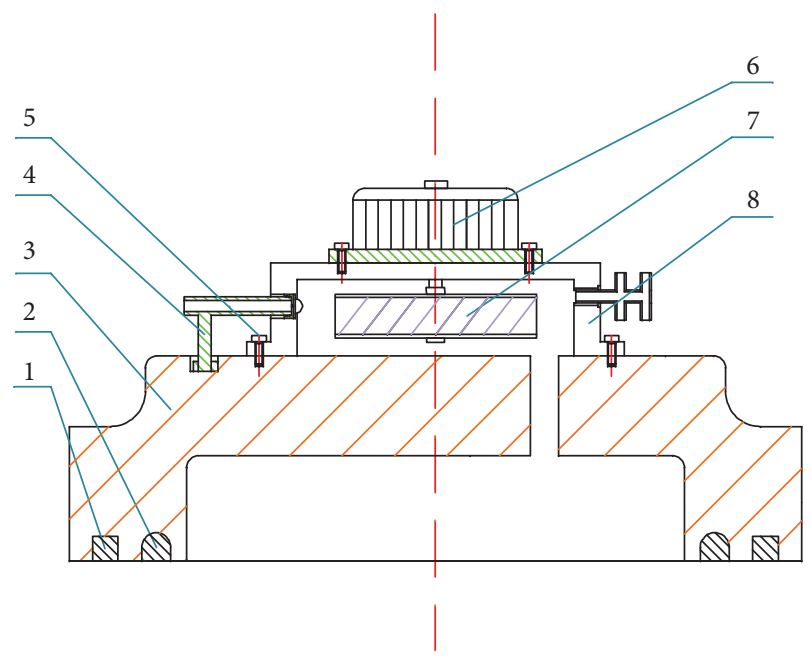

FIGURE 7: Negative pressure adsorption mechanism of intelligent safety monitoring robot for shaft construction. (1) Sealing ring 1; (2) sealing ring 2; (3) adsorption disc; (4) air outlet; (5) set bolt; (6) adsorption motor; (7) impeller; (8) shell.

With the increase of the input voltage of the driving motor of the centrifugal fan, the power consumption of the negative pressure generator, and the difference between negative pressure inside and outside the negative pressure chamber grew accordingly. Under the same input voltage, the negative pressure generator needed to consume more power when the inlet of the glass rotameter was fully opened. If the sealing effect of the robot can be improved and the air leakage can be further reduced, the power consumed by the fan can be decreased dramatically. As far as the robot was concerned, its working time was obviously prolonged when carrying the same power supply.

The product of the flow rate and negative pressure of the negative pressure generator, divided by the power consumption of the driving motor, was the efficiency of the negative pressure generator. The efficiency of the negative pressure generator of the wall-climbing robot during the variation of air leakage flow was analyzed, and the results are shown in Figure 9(a). When the wall-climbing robot ran on different walls, due to different roughness of the wall surfaces, the leakage flow of the sealing mechanism changed. The negative pressure generated by the negative pressure generator at different flow rates is displayed in Figure 9(b).

It can be seen from Figure 9(a) that when the flow rate of the negative pressure generator was greater than $30 \mathrm{~m}^{3} /$ hour and less than $75 \mathrm{~m}^{3} /$ hour, its efficiency was close to or greater than $90 \%$, and the fan showed high efficiency as a whole, which was beneficial to the reduction of noise radiation of the wall-climbing robot.

As shown in Figure 9(b), within a large variation range of flow rate, the negative pressure output by the negative pressure generator showed straight flow rate-negative pressure features. When the robot encountered a change in the wall crack, the air leakage of the sealing mechanism changed, but the centrifugal fan can still output stable negative pressure, which offered a guarantee for the stable adsorption of the wall-climbing robot and satisfied the robot's requirements of safe adsorption.

4.4. Robot Control and Drive Design. The robot control module is the drive control and function control parts of the robot, which can implement automatic crawling, probe scanning movement, and its control. The master control module is responsible for the coordination and external interaction of such modules as human-computer interaction display, sensor acquisition, visual processing, drive control, and communication modules in the whole system. As shown in Figure 10, the module consists of a master control embedded control unit, master control embedded processing unit, and wireless data transmission module. The design of a portable handheld control box greatly reduces the operation difficulty and can display the site images and sound in real time. The remote control handle can control the movement of the robot. The robot also has a remote control function, which is operated by the operator with the computer at the ground control center. The robot can patrol by itself according to the set planning route. Aiming at the operating characteristics of the intelligent safety monitoring robot for shaft construction, the mainstream technology of existing wall-climbing robots and separate study on the motion system control and the adsorption system control as two independent parts are changed, and the two are combined for research to achieve the optional reliable control of the motion system and the adsorption system.

The driving motor of the robot is a brushless DC servo motor, with the advantages of small motor size, lightweight, large output force, quick response, high speed, small inertia, smooth rotation, stable moment, and intelligence. Besides, its electronic commutation mode is flexible, including square wave commutation or sine wave commutation. The motor also has the characteristics of no need of replacing the carbon brush, maintenance-free, high efficiency, low operating temperature, low noise, low electromagnetic radiation, and long life, which is applicable to the coal mine shaft environment. 


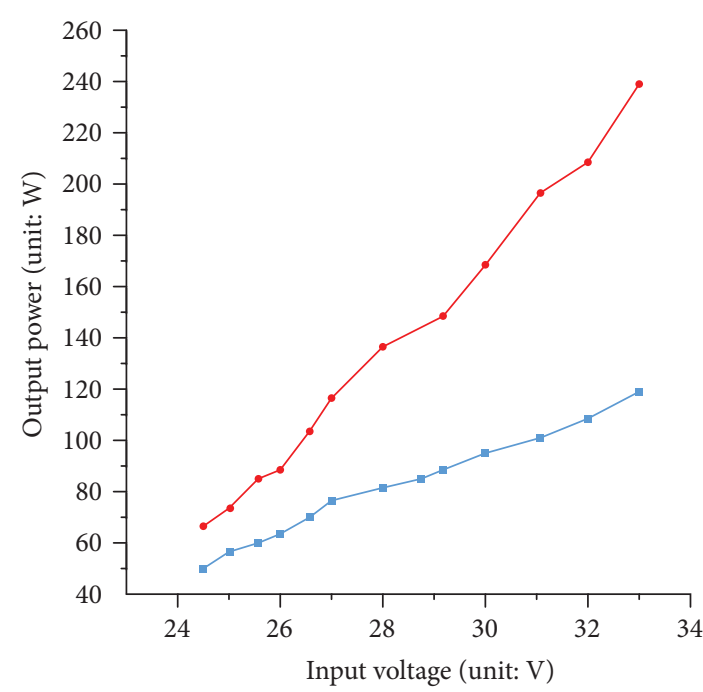

(a)

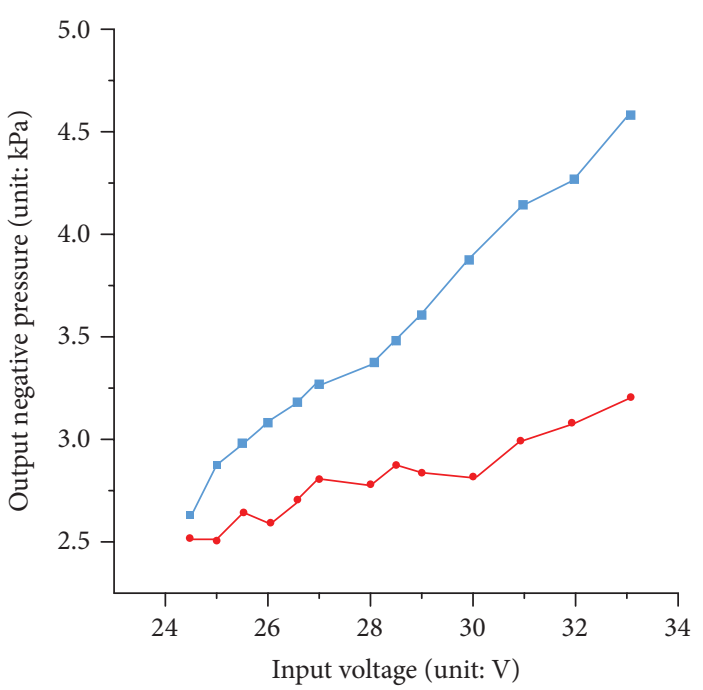

(b)

Figure 8: Pressure generator performance under different inlet situations. (a) Comparison of output power. (b) Comparison of output pressure.

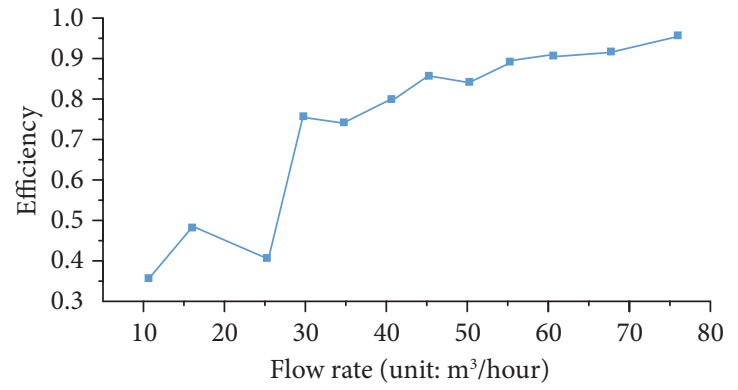

(a)

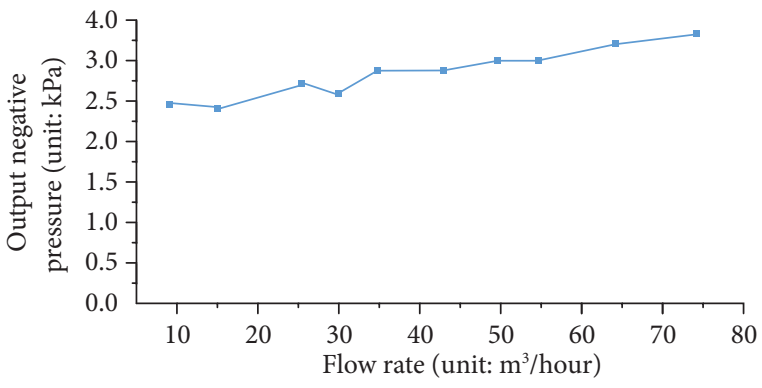

(b)

FIGURE 9: Efficiency and pressure output of pressure generator. (a) Efficiency of pressure generator. (b) Pressure output of pressure generator.

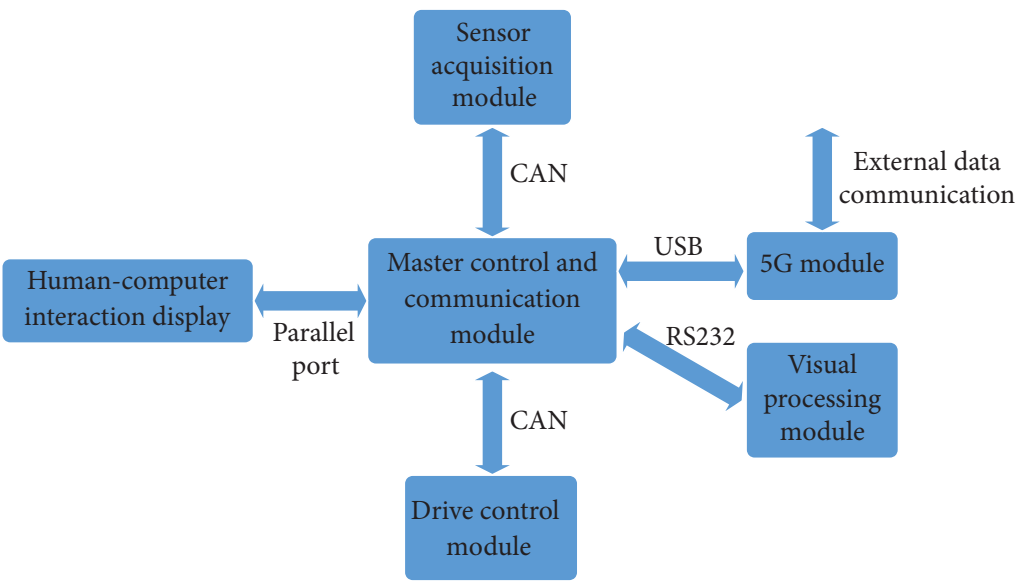

Figure 10: Control module of intelligent safety monitoring robot for shaft construction. 
To implement the autonomous walking patrol of the robot, it is necessary to design the robot servo motor control system and position determination module. The servo controller is a controller used to control the servo motor, and its function is similar to that of the frequency converter on the ordinary AC motor. The servo controller is an important part of the servo system, and it is mainly used in the highprecision position control system. Generally, the servo motor is controlled by position, speed, and torque to implement the precise positioning of the transmission system. The servo driver is designed to adopt the mainstream digital signal processor (DSP) as the control core, which can realize a complex optimization control algorithm and give consideration of the digitalization, networking, and intelligence at the same time. The driving circuit of the power device is designed with the intelligent power control module (IPM) as the core, and the control circuit function and high power electronic switching device are integrated into the IPM. It has input isolation, energy consumption braking, overtemperature, overvoltage, over current protection, intelligent fault diagnosis, and other functions. The soft starting circuit is also designed in the main circuit to reduce the impact of the motor starting process on the driver. The power driving unit first rectifies through the full-bridge rectifier circuit to get the corresponding direct current and then drives the servo motor through the conversion of the PWM inverter frequency. Since the precision of open-loop control cannot well meet the requirements of the robot, the most fundamental method is to adopt the closed-loop control mode in order to improve the control precision of the servo drive. In other words, there is not only a precursor control channel but also a feedback channel to detect the output. The deviation signal is obtained after the comparison between the instruction signal and the feedback signal to form the closed-loop control system with the deviation control.

The motion control algorithm of the safety monitoring robot walking along the shaft is the key to the robot design. The control algorithm can ensure that the robot runs smoothly along the set walking path, reaches the expected posture and patrol speed, and can adapt to the strong electromagnetic interference environment in the shaft. The control function is mainly implemented by the feedback of the position loop, speed loop, and current loop of the robot motion control system. The so-called loop refers to providing feedback to the processing link for the enhancement of system stability and performance. The speed loop is established based on the current loop, and the position loop is established based on the speed loop and the current loop. The input of the position loop specifically refers to the external pulse, which is processed by smoothing filtering and electronic calculation as the setting of the "position loop." The setting and the pulse signal feedback from the encoder, after calculation by the deviation counter and the PID adjustment at the position loop, are output. Together with the feedforward signal given by the position, they are input to the speed loop. Then, the difference after the comparison between the input value and the "speed loop feedback" value in the speed loop is input to the current loop as the value after PID adjustment. The difference after the comparison between the input value and the "current loop feedback" value in the current loop is output to the motor as the value after PID adjustment. The "output of current loop" is the phase current of the motor at each phase. Asynchronous sampling is used to design the position loop and speed loop. The sampling cycle of the position loop is set to $T_{1}=5 \mathrm{~ms}$ and that of the speed loop is set to $T_{2}=128 \mu \mathrm{s}$. The current loop is designed to select the parameters of the power control module reasonably. Generally, the adjustment of the position loop is to control different algorithms of the curvilinear motion of the mobile robot. Kanayama proposed a simple and effective control algorithm based on the kinematics model of the mobile robot, which can guarantee stable trajectory tracking characteristics. The correct and practical design of the path walking control algorithm of the safety monitoring robot is reflected in the asymptotic convergence of the robot walking track to the planned inspection path, posture, and speed, which are consistent with the planning requirements. $(x, y, \theta)^{T}$ in the Cartesian coordinate system represents the actual posture of the robot. $(x, y, \widetilde{\theta})^{T}$ is the error between the actual posture $(x, y, \theta)^{T}$ of the robot and the planned posture $\left(x_{r}, y_{r}, \theta_{r}\right)^{T} . v$ and $w$ are the translational and rotational speeds of the robot during walking, and they are the planned speed. The objective of the control algorithm is to seek the feed control rate $U=$ $\left(u_{1}+u_{2}\right)^{T}$ so that for any initial posture and speed error, the robot has the following under the control algorithm:

$$
\lim _{1 \longrightarrow \infty}\left|\left(X, Y, \tilde{\theta}, v-v_{r}, w-w_{r}\right)^{T}\right|=0 .
$$

When the robot tracks the track of the planned route, the error equation is described as

$$
\left[\begin{array}{c}
\dot{X} \\
\dot{Y} \\
\dot{\tilde{\theta}}
\end{array}\right]=\left[\begin{array}{c}
-v+Y w+v_{r} \cos \tilde{\theta} \\
-X w+v_{r} \sin \tilde{\theta} \\
w_{r}-w
\end{array}\right] .
$$

The stable control algorithm is designed as follows:

$$
\begin{aligned}
U & =\left[\begin{array}{l}
u_{1} \\
u_{2}
\end{array}\right] \\
& =\left[\begin{array}{c}
\dot{v}_{0}-\lambda\left(v-v_{0}\right) \\
\dot{w}_{0}-\lambda\left(w-w_{0}\right)
\end{array}\right],
\end{aligned}
$$

where

$$
\left[\begin{array}{c}
v_{0} \\
w_{0}
\end{array}\right]=\left[\begin{array}{c}
v_{r} \cos \tilde{\theta}+K_{X} X \\
w_{r}+v_{r}\left(K_{Y} Y+K_{\theta} \sin \tilde{\theta}\right)
\end{array}\right] .
$$

Formula (11) shows the control algorithm proposed by Kanayama directly considering the kinematics model of the robot, where translational velocity $v$ and rotational velocity $w$ of the robot are control variables, and $K_{X}, K_{Y}$, and $K_{\theta}$ are bounded parameters: 


$$
\lambda=\frac{\left[\dot{V}_{0}+\left(\dot{V}_{0}^{2}+b^{2}\right)^{1 / 2}\right]}{b},
$$

where $\lambda \geq 0$ is dynamic convergence gain:

$$
\begin{gathered}
\dot{V}_{0}=-K_{X} X^{2}-\frac{v_{r} K_{\theta} \sin ^{2} \tilde{\theta}}{K_{y}}, \\
b=\left(v-v_{0}\right)^{2}+\left(w-w_{0}\right)^{2} .
\end{gathered}
$$

Based on the above formulas, the control algorithm is a function of the state variable $(X, Y, \widetilde{\theta}, v, w)^{T}$. Since it is based on the dynamics model of the robot and the algorithm is the feedback control of moment, $\left(u_{1}, u_{2}\right)^{T}=(v, w)^{T}$. Under the action of control algorithm (10), the five-dimensional state variable $(X, Y, \widetilde{\theta}, v, w)^{T}$ of the mobile robot asymptotically converges to $\left(\varepsilon_{1}, \varepsilon_{2}, \varepsilon_{3}, v_{r}, w_{r}\right)^{T}$, where $\varepsilon_{1}, \varepsilon_{2}$, and $\varepsilon_{3}$ are small enough. In other words, when the algorithm of formula (10) is used to control the safety monitoring robot to walk along the planned route, the position error between the actual running track of the safety monitoring robot and the planned route will converge to a small enough value, and the actual walking speed of the safety monitoring robot can also reach the planned speed set when walking according to the planned route.

4.5. Robot Communication Design. The robot communication system is an important means for the robot to implement information exchange. In the aspect of robot communication design, with the increasing complexity of information processing, different robot communication methods are selected according to the needs of the working environment and different application fields. It is necessary to study the communication technology and method of the safety monitoring robot for coal mine shaft according to the structural characteristics of the shaft. Due to the narrow and long space of the shaft and the large depth of the coal mine shaft, the traditional wired communication method has some shortcomings, such as the inability to match the robot and the difficulty in laying cables in the shaft. Hence, the optimal scheme is to adopt the wireless communication method and study the wireless coverage technology of coal mine shafts. In order to ensure the real-time transmission of robot data and control signals, the wireless network coverage technology for coal mine shaft construction period based on $5 \mathrm{G}$ is studied. The system is composed of $5 \mathrm{G}$ core network, $5 \mathrm{G}$ base station controller, $5 \mathrm{G}$ base station, and $5 \mathrm{G}$ industrial router. The wireless base station for $5 \mathrm{G}$ communication is set at an appropriate position in the shaft to realize $5 \mathrm{G}$ signal coverage of the shaft. $5 \mathrm{G}$ industrial module is designed and installed on the robot body to realize realtime control and high-speed data transmission of the robot. As the core component of $5 \mathrm{G}$ network connection for industrial products, $5 \mathrm{G}$ industrial module encapsulates $5 \mathrm{G}$ baseband chips, RF, storage, power management, and other hardware together and provides standard hardware and software interfaces to lower the threshold for industry users.
The encapsulated modules also reduce the overall cost of the system. The use environment of industrial modules is complex and harsh. 5G industrial modules are required not only to have high speed and low delay but also to be high and low temperature resistant, more reliable, and safer. They also should have the ability of network-oriented evolution to protect the equipment. The industrial module is designed to adopt the Huawei Balong HM5000-31 chip, and the core components include master chip, PMU, and RF devices, so it is applicable to the safety monitoring rotor for shafts. NSA/SA dual mode is supported, and different 5G mode networks can be connected flexibly according to actual needs. 5G SA 5G SA and 2G/3G/4G/5G compatibility are supported for direct synchronization of $5 \mathrm{G}$ network coverage. The download rate is as high as $2 \mathrm{Gbps}$, and the upload rate is as high as $230 \mathrm{Mbps}$, meeting the high bandwidth requirements of shaft safety inspection. The operating temperature range is $-40^{\circ} \mathrm{C}-85^{\circ} \mathrm{C}$, and high-reliability devices and unique industrial designs are adopted to adapt to the complex environment of coal mine shafts. The dual safety mechanism of TrustZone supported by the microkernel can ensure the safety monitoring robot works safely and reliably. With the high-performance application processor, its computing power is up to 14400DMPIS, and 18 types of hardware interfaces can fully meet the needs of industrial equipment interface.

4.6. Intelligent Sensing System Design of the Robot. The selection of robot sensors depends on the working needs and application characteristics of the robot. For the complexity of the environment in the period of shaft construction, especially the diversity of gas components in the shaft environment, it is necessary to study the monitoring and sensing technology of the shaft environment. The curves of shaft sinking speed and methane concentration are shown in Figure 11.

Firstly, it is necessary to study the sensing technology suitable for the environment of the coal mine shaft, develop intelligent sensors, develop a wind sensor for real-time monitoring of shaft wind speed, develop a methane sensor to monitor methane gas concentrations in the shaft environment, develop a carbon monoxide sensor to monitor carbon monoxide gas concentrations in the shaft environment, develop a dust sensor to monitor dust concentration in the shaft, and develop a smoke sensor to monitor the construction environment after blasting. In addition, the data acquisition device can communicate with the sensor in real time to collect and receive the data information of the sensor and achieve collection and monitoring of shaft environment information. Different weight grades are distributed to the data collected by different sensors and different locations according to safety prewarning needs and data importance degree. Fusion calculation and result evaluation of several monitoring data is performed using a fuzzy comprehensive evaluation model. By doing so, it makes up for the deficiency that one-sided data cannot fully reflect the mine safety level, thereby realizing accurate monitoring and objective evaluation of the overall safety of 


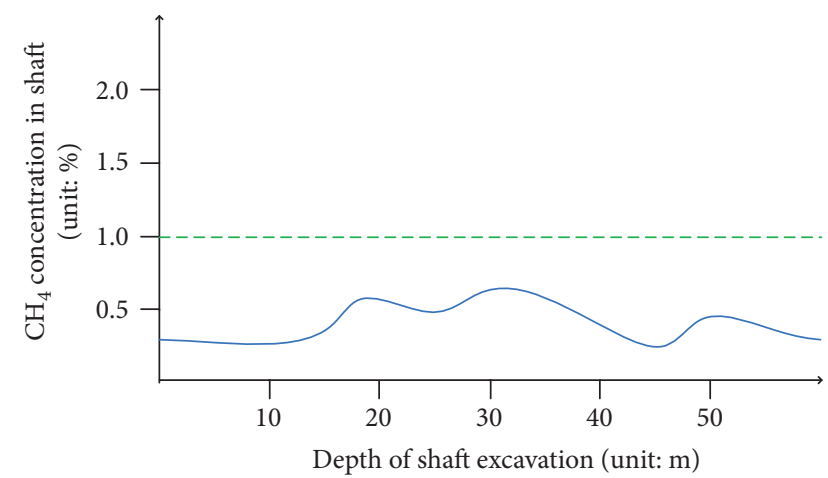

Figure 11: The curves of shaft sinking speed and methane concentration.

the whole mine shaft area. The structure of the data acquisition device is shown in Figure 12. Moreover, to achieve autonomous walking and obstacle avoidance of the robot, the robot also should be equipped with IMU posture sensor, laser radar obstacle avoidance sensor, ultrasonic ranging obstacle avoidance sensor, and depth camera sensor. The sensor acquisition module uses STM32F407 SCM as the core of the module acquisition, conducts communication and data acquisition with various sensors through different interfaces, and packs and uploads the data to the master control and communication module after summarization. This design is easy to expand and reduce the pressure of the master control module.

4.7. Design of Hazard Source Recognition System. The sensing system is the most important part of the interaction of the intelligent robot with the human and external environment. Due to the complex environment of the shaft construction period, it is difficult for production and operation personnel to inspect. Due to blasting, external force, and groundwater, the safety monitoring problem for shafts stands out, which is sudden and unpredictable. In case of any abnormal condition, relevant personnel cannot be notified in time, which may easily cause safety accidents. Hence, the intelligent safety monitoring robot for coal mine shaft construction shall have the ability of visual recognition of hidden dangers. Visual recognition technology, also known as image recognition technology, can replace manpower to realize image recognition and classification by means of digital image processing and computer high-performance computing. By using visual recognition technology, image content can be quickly recognized through image collection, preprocessing, feature collection, classification decision-making, and other functions. An actual on-site condition can be accurately judged. By providing standardized supporting data, together with the automatic control system, it can execute a specific operation according to the set program.

The visual recognition unit of the intelligent safety monitoring robot system is based on TCP/IP and SIP protocols. The advanced digital signal processing technique can implement video data acquisition (developing video capture device and recognition technology). It has intelligent analysis (able to distinguish abnormal situations and faults of shafts) function. The intelligent software platform can be linked with the intelligent broadcasting system to broadcast safety warning information so that safety supervision personnel can grasp the state of the shaft in time. The professional video capture device can overcome the interference of video recognition caused by shaft lighting limit, lack of color information, and similar target and background. The system applies video enhancement algorithm and intelligent video recognition technology, which can detect the abnormal state information of "man-machine-environment" from the video image of a dangerous area, automatically capture and track the abnormal point, discover in time and intelligently identify shaft wall fracture, water seepage, and other dangerous sources, as well as empty roof operation and other violations, and give timely warning. It can also be integrated with other shaft monitoring systems to identify potential safety hazards such as structural stress change of shaft wall and shaft wall deformation and provide the site basis for technical personnel to evaluate the safety state of the shaft and make the decision of shaft construction, which can effectively guarantee the safety of shaft construction and improve the efficiency of shaft construction. As well, the system can intelligently judge and track autonomously the abnormal position and give early warning in time. The functions of the hazard source recognition module are shown in Figure 13.

4.8. Design of Miss-Fire Explosive Recognition System. In coal mine shaft construction, blasting construction is a common working mode. In the process of blasting construction, due to the quality of the explosive, the quality of the detonator, the damage of the gun wire, the bad contact of the gun wire, and other reasons, individual explosive detonators fail. The miss-fire explosives remain in the muck. In the muck cleaning and transportation process, explosives may explode due to the impact of external forces. There is an unpredictable risk of explosion, and this can also cause casualties and property damage. Miss-fire explosive detection is a difficulty of current blasting operation. Manual detection has the disadvantage of high risk and low recognition rate. The automatic recognition device for missfire explosive can be carried by the robot to ensure the speed and reliability of information reading. The wireless communication technology and flexible label coding identification technology can be used to reliably identify the miss-fire detonator and explosive, which greatly improves the detection rate of miss-fire explosives. The recognition work does not require manual interference, and automatic recognition and warning can be implemented. After blasting, the robot can detect the miss-fire explosive and safe state of the operating plane. After confirming the safety, workers can enter the construction site. Such practice greatly safeguards the safety of operating personnel and reduces accidents.

The intelligent recognition device of miss-fire detonators and miss-fire explosives in shaft construction can accurately recognize miss-fire detonators and miss-fire explosives in the blasting construction during the coal mine blasting 


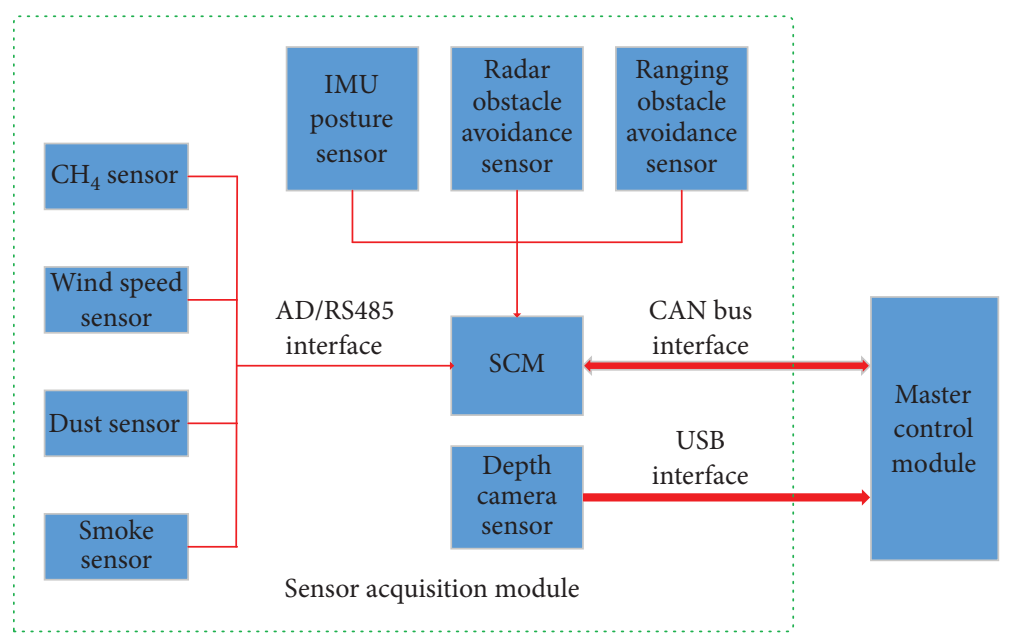

FigURE 12: Structure of sensor acquisition module.

period. The intelligent recognition device of miss-fire detonators and miss-fire explosives in shaft construction is mainly composed of a portable recognition host of miss-fire detonators and miss-fire explosives and flexible recognition label. As shown in Figure 14, the portable recognition host of miss-fire detonators and miss-fire explosives mainly consists of shell, data interface, sound alarm device, alarm module, portable device, LED display module, light alarm device, communication module, antenna, control panel, mainboard, and battery module. The flexible recognition label is composed of a flexible pastable body, chip mainboard, and external identification.

The wireless communication technology and flexible label coding recognition technology are applied in the intelligent recognition device of miss-fire detonators and missfire explosives in shaft construction. A passive electronic radio frequency tag with frequency hopping operating mode is designed, which has a very strong antijamming capability. With more accurate and faster recognition capacity, the noncontact reading mode is realized, and the distance can reach more than $10 \mathrm{~m}$. The device can read the information of multiple explosives and detonators at the same time, with ultralow power consumption. The size and weight decrease effectively, and the device can be arranged flexibly and safely. The speed and reliability of information reading can be ensured, and the position and quantity of miss-fire detonators and explosives can be accurately and reliably identified. The plane position of detonator monitoring is shown in Figure 15.

Prior to blasting, each detonator and each explosive cartridge are fixed on the passive flexible recognition label, and coding and position setting are conducted. The portable recognition host of miss-fire detonators and explosives and the flexible recognition label can keep wireless real-time communication and receive and send state information data. After shaft blasting, the portable recognition host of missfire detonators and explosives can be carried by the safety monitoring robot or safety monitoring personnel to accurately distinguish miss-fire detonators and miss-fire explosives.

\section{Design of Robot Software Platform}

The robot software platform can support all functions of the robot, and its development quality concerns the success or failure of robot research. For the problems of high specificity, low developability, and high upgrade cost of robot control software, the general control software platform scheme is proposed. The general robot software platform can display the video pictures sent back by the robot in real time, display hazardous gas data collected by the robot, alarm for abnormal data, and analyze, store, share, and print data. Besides, the platform has the function of remote control and remote parameter setting for the robot, which can assist the robot in implementing intelligent decision-making and provide technical support for safety managers to make correct decisions. The platform has the following functions: making and issuing robot inspection plan, implementing $24 \mathrm{~h}$ patrol of the robot, remote parameter setting, remote control of robot, robot real-time state monitoring, processing and display of shaft information collected by the robot, robot state display, visual intelligent identification of hazard sources in shaft construction, intelligent warning, data storage, relevant system data access, and report printing. The real-time data of working face distance, wind speed, and dust concentration are shown in Figure 16.

Considering the platform development, visualization requirements, interface, and communication requirements, compared with Windows operating system, the Linux visualization engine (Unity) is difficult to deploy and maintain. In addition, it is necessary to convert to $\mathrm{C}++$, and some functions of $\mathrm{C} \#$ cannot be implemented. Moreover, it is also difficult to maintain the network, serial port, and other peripheral sensing nodes under Linux. Hence, Windows 10 is chosen as the operating system. Unity (version no. 2018.4.8f1) is chosen as the standard for the master software interface development platform. This version is a long-supported version of Unity (LTS), which is beneficial to subsequent maintenance. Compared with the development platform based on graphics components such as QT, Unity has such advantages as saving development cost, being based on 


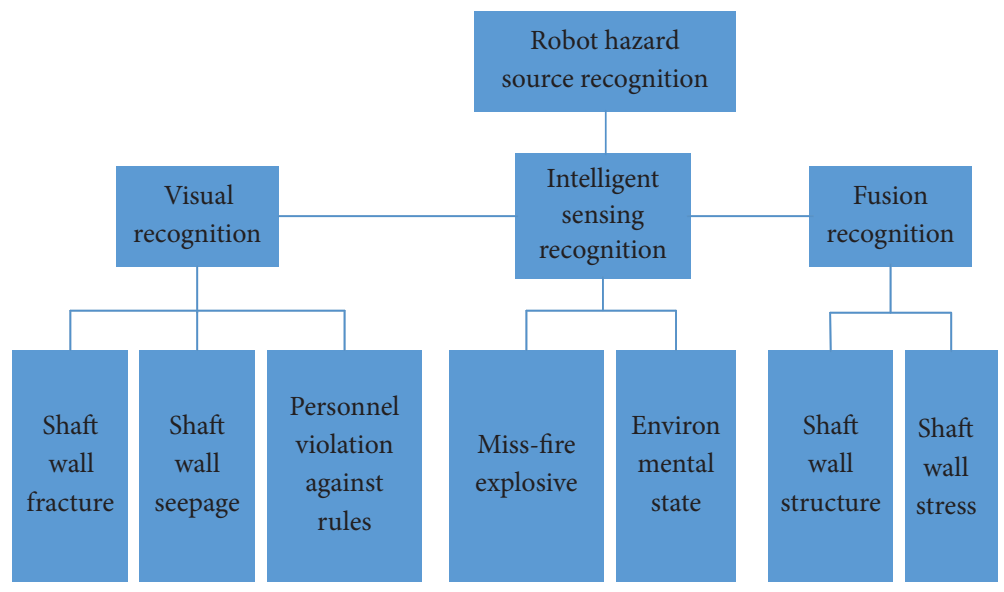

Figure 13: Functions of hazard source recognition module.

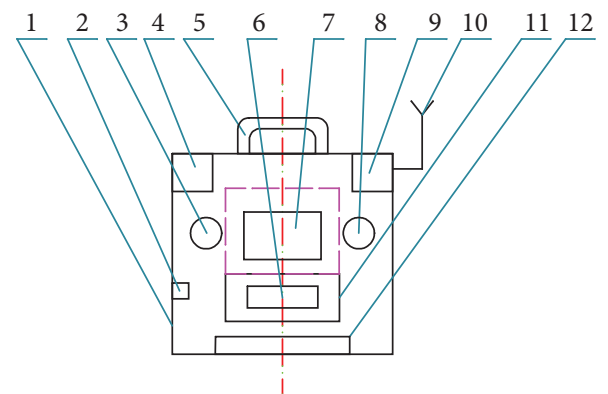

(a)

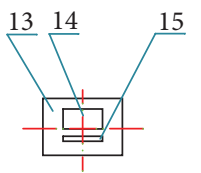

(b)

FIgURE 14: Intelligent recognition device of miss-fire detonators and miss-fire explosives in shaft construction. (a) Portable recognition host of miss-fire detonators and miss-fire explosives; (b) flexible recognition label. (1) Shell; (2) data interface; (3) sound alarm device; (4) alarm module; (5) portable device; (6) control panel; (7) LED display module; (8) light alarm module; (9) communication module; (10) antenna; (11) mainboard; (12) battery module; (13) flexible pastable body; (14) chip motherboard; (15) external identification.

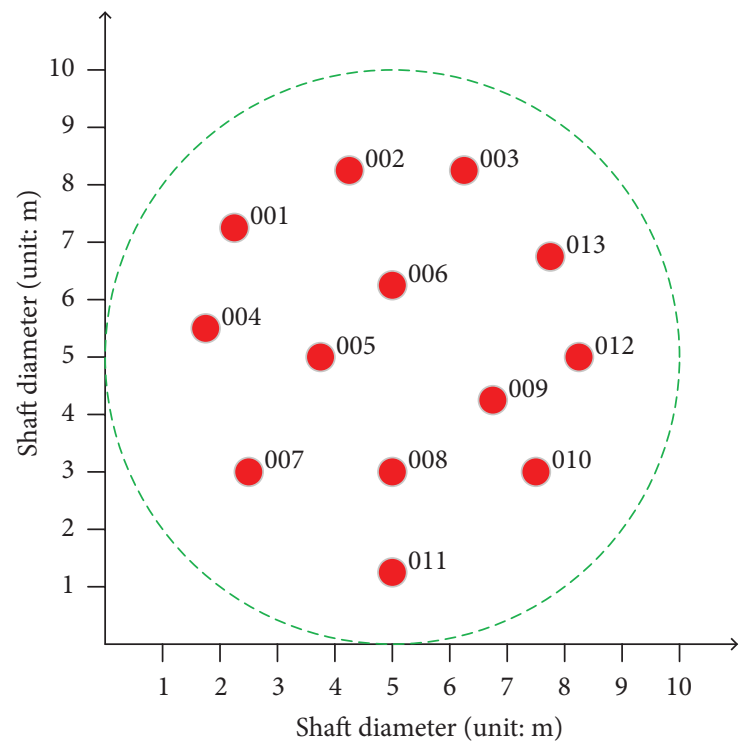

FIGURE 15: The plane position of detonator monitoring. 


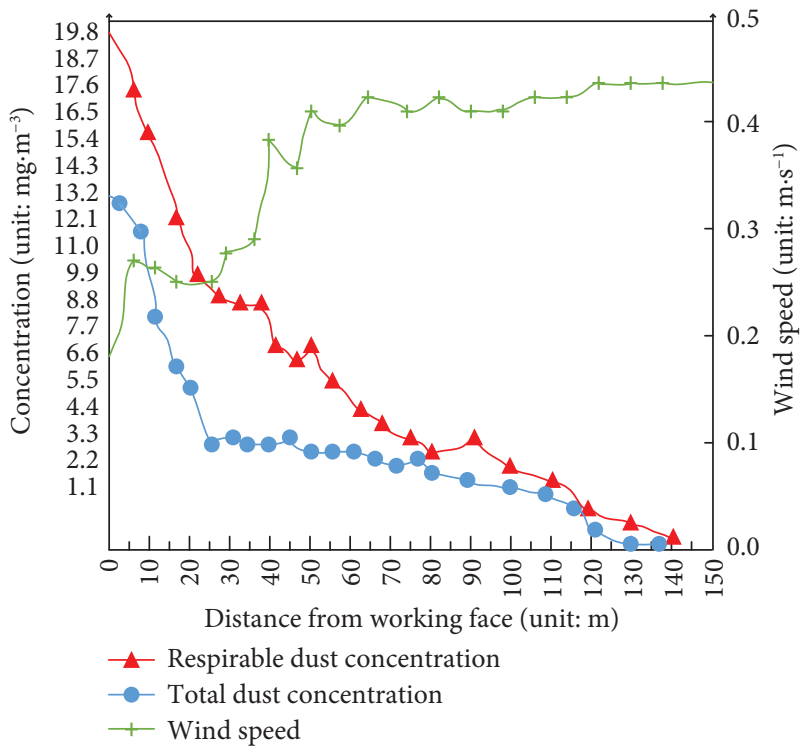

FIGURE 16: The real-time data of working face distance, wind speed, and dust concentration.

components and frame, high rendering performance, advanced light effect particle system, and extensibility.

\section{Conclusion}

This study discusses the design and research of intelligent safety monitoring robot during the construction of coal mine shaft from the perspective of practical application. The research project can effectively reduce all kinds of accidents during the construction of the coal mine shaft. The organic combination with the equipment and personnel management system better plays the role of the safety monitoring robot, guarantees work safety in the period of shaft construction, improves shaft construction efficiency, reduces personnel and property loss, and saves human resource cost. Because the intelligent safety monitoring robot technology for coal mine shaft construction has no successful precedent for reference, this study has certain technical limitations. The robot's functions and performance indexes still remain to be improved and perfected. The research of coal mine safety monitoring robot just starts. As national coal mines become intelligent and the unmanned strategy is gradually advanced, the coal mine safety monitoring robot will play a greater role in the field of coal mine safety inspection, and the technical development of coal mine safety monitoring robot will change quickly. With the promotion and application of safety monitoring robot in the shaft construction period, safety hazards can be found in time and faults can be cleared. Meanwhile, safety guarantee capability in the shaft construction period can improve, and safety accidents in the shaft construction period will certainly be reduced. Therefore, the smooth implementation of coal mine construction is guaranteed.

\section{Data Availability}

No data were used to support this study.

\section{Conflicts of Interest}

The authors declare that they have no conflicts of interest.

\section{Authors' Contributions}

All authors have read and approved the manuscript.

\section{References}

[1] Y. Zhao, Theory and Technology of Mine Dust Prevention, pp. 2-3, Coal Industry Press, Beijing, China, 1995.

[2] B. T. Le, R. J. Goodey, and S. Divall, "Subsurface ground movements due to circular shaft construction," Soils and Foundations, vol. 59, no. 5, pp. 1160-1171, 2019.

[3] T. Schwamb, M. Elshafie, K. Soga, and R. Mair, "Considerations for monitoring of deep circular excavations," Proceedings of the Institution of Civil Engineers-Geotechnical Engineering, vol. 169, p. 6, 2016.

[4] D. F. Malan, "Manuel rocha medal recipient simulating the time-dependent behaviour of excavations in hard rock," Rock Mechanics and Rock Engineering, vol. 35, no. 4, pp. 225-224, 2002.

[5] D. F. Malan, "Time-dependent behaviour of deep level tabular excavations in hard rock," Rock Mechanics and Rock Engineering, vol. 32, no. 2, pp. 123-155, 1999.

[6] V. D. Baloyi and L. D. Meyer, "The development of a mining method selection model through a detailed assessment of multi-criteria decision methods," Results in Engineering, vol. $8,2020$.

[7] F. Wang, T. Ren, S. Tu, H. Frank, and N. Aziz, "Implementation of underground longhole directional drilling technology for greenhouse gas mitigation in Chinese coal mines," International Journal of Greenhouse Gas Control, vol. 11, pp. 290-303, 2012.

[8] S. Bhattacharjee, P. Roy, S. Ghosh, S. Misra, and M. S. Obaidat, "Wireless sensor network-based fire detection, alarming, monitoring and prevention system for Bord-andPillar coal mines," Journal of Systems and Software, vol. 85, pp. 571-581, 2012. 
[9] W. Qiao, "Analysis and measurement of multifactor risk in underground coal mine accidents based on coupling theory," Reliability Engineering and System Safety, vol. 208, 2012.

[10] M. Spada and P. Burgherr, "An aftermath analysis of the 2014 coal mine accident in soma, turkey: use of risk performance indicators based on historical experience," Accident Analysis and Prevention, vol. 87, 2016.

[11] M. A. Bauer, V. A. Dmitrienko, and A. I. Kapustin, "Assessment of deformations of Earth's surface at mine construction on sub-soils," Procedia Engineering, vol. 150, 2016.

[12] J. Zhang, J. Fu, H. Hongyu, G. Fu, F. Nie, and W. Zhang, "Root causes of coal mine accidents: characteristics of safety culture deficiencies based on accident statistics," Process Safety and Environmental Protection, vol. 136, 2020.

[13] J. Zhang, K. Xu, R. Genserik, and G. You, "Statistical analysis the characteristics of extraordinarily severe coal mine accidents (ESCMAs in China from 1950 to 2018)," Process Safety and Environmental Protection, vol. 133, 2020.

[14] Q. Shao, Z. Zhang, and Z. Zhang, "Research on safety monitoring system of mine based on ad hoc technology," in Proceedings of the International Conference on Information Management, Innovation Management and Industrial Engineering, Xi'an, China, November 2013.

[15] Y. Zhang, W. Yang, D. Han, and Y.-I. Kim, “An integrated environment monitoring system for underground coal mines-wireless sensor network subsystem with multi-parameter monitoring," Sensors, vol. 14, Article ID 13149, 2014.

[16] C. Ö. Karacan, "Modeling and prediction of ventilation methane emissions of US longwall mines using supervised artificial neural networks," International Journal of Coal Geology, vol. 73, pp. 371-387, 2008.

[17] C. Ö. Karacan, "Forecasting gob gas venthole production performances using intelligent computing methods for optimum methane control in longwall coal mines," International Journal of Coal Geology, vol. 79, pp. 131-144, 2009.

[18] J. Edwards, R. Franks, G. Friel, C. Lazzara, and J. Opferman, "Real-time neural network application to mine fire-nuisance emissions discrimination," in Proceedings of the 10th US/ North American Mine Ventilation Symposium, Anchorage, AK, USA, May 2004.

[19] J. Edwards, G. Friel, R. Franks, C. Lazzara, and J. Opferman, "Mine fire source discrimination using fire sensors and neural network analysis," in Proceedings of the 2000 Technical Meeting of Central States Section of the Combustion Institute, Indianapolis, IN, USA, April 2000.

[20] X. Ma and H. Zhu, "Gas concentration prediction based on the measured data of a coal mine rescue robot," Journal of Robotics, vol. 2016, Article ID 6858970, 10 pages, 2016.

[21] L. Ma and Q. Chen, "Problems and research on underground charging safety of power battery for coal mine robot," IOP Conference Series: Earth and Environmental Science, vol. 651, no. 3, 2021.

[22] G. Zhou, L. Huang, Z. Zhu, W. Li, and G. Shen, “A zoning strategy for uniform deployed chain-type wireless sensor network in underground coal mine tunnel," in Proceedings of the 2013 IEEE 10th International Conference on High Performance Computing and Communications \& 2013 IEEE International Conference on Embedded and Ubiquitous Computing (HPCC_EUC), Zhangjiajie, China, November 2013.

[23] W. Farjow, K. Raahemifar, and X. Fernando, "Novel wireless channels characterization model for underground mines' applied mathematical modeling," Applied Mathematical Modelling, vol. 39, pp. 5997-6007, 2015.
[24] B. W. Jo and R. M. A. Khan, "An event reporting and earlywarning safety system based on the internet of things for underground coal mines: a case study," Applied Sciences, vol. 7, p. 925, 2017.

[25] U. I. Minhas, I. H. Naqvi, S. Qaisar, K. Ali, S. Shahid, and M. A. Aslam, "A WSN for monitoring and event reporting in underground mine environments," IEEE Systems Journals, vol. 12, pp. 485-496, 2017.

[26] S. Masataka, K. Shinya, and H. Shigeo, "Basic systematic experiments and new type child unit of anchor climber: awarm type wall climbing robot system," in Proceedings of IEEE International Conference on Robotics and Automation, Pasadena, TX, USA, May 2008.

[27] J. Shang, B. Bridge, T. Sattar et al., "Development of a climbing robot for inspection of long weld lines," Industrial Robot: International Journal, vol. 35, no. 3, pp. 217-223, 2008.

[28] L. P. Kalra and J. Gu, "An autonomous self contained wall climbing robot for non-destructive inspection of aboveground storage tanks," Industrial Robot: International Journal, vol. 34, no. 2, pp. 122-127, 2007.

[29] A. Keisuke and H. Shigeo, "Study of walking robot for 3 dimensional terrain," in Proceedings of IEEE International Conference on Robotics and Automation, Nagoya, Japan, May 1995.

[30] H. Zhang, J. Zhang, G. Zong, W. Wang, and R. Liu, "Sky cleaner 3: a real pneumatic climbing robot for glass-wall cleaning," IEEE Robotics and Automation Magazine, vol. 13, no. 1, pp. 32-41, 2006.

[31] M. Dave, "Potential use of walking and climbing robots in nuclear facilities," Nuclear Engineer, vol. 42, no. 1, pp. 4-8, 2001.

[32] N. Elkmann, T. Felsch, M. Sack, T. Boehme, J. Hortig, and J. Saenz, "Modular climbing robot for service-sector applications," Industrial Robot, vol. 26, no. 6, pp. 460-465, 1999.

[33] D. Xu, S. Liu, D. Zhou, and Y. Wang, "Design of a wall cleaning robot with a single suction cup," in Proceedings of the Second International Conference CLAWAR, Portsmouth, UK, 1999.

[34] T. Yano, T. Suwa, M. Murakami, and Yamamoto, "Development of a semi self-contained wall climbing robot with scanning type suction cups," in Proceedings of IEEE International Conference on Intelligent Robots and Systems, Grenoble, France, September 1997.

[35] S. Kim, M. Spenko, S. Trujillo et al., "Smooth vertical surface climbing with directional adhesion," IEEE Transactions on Robotics, vol. 24, no. 1, pp. 65-74, 2008.

[36] B. Zhao, N. Pesika, K. Rosenberg et al., "Adhesion and friction force coupling of gecko setal arrays: implications for structured adhesive surfaces," Langmuir, vol. 24, no. 4, pp. 1517-1524, 2008.

[37] N. Akihiko and H. Shigeo, "Walking and running of the quadruped wall-climbing robot," in Proceedings of IEEE International Conference on Robotics and Automation, San Diego, CA, USA, May 1994.

[38] S. Hirose and K. Kawabe, "Ceiling walk of quadruped wall climbing robot NINJA-II," in Proceedings of the First International Conference CLAWAR 98, Bury St Edmunds: Professional Engineering Publishing, Brussels, Belgium, November 1998.

[39] P. Mansi and L. P. Pratap, "Advancement of a wall climbing robot for various applications," International Journal of Engineering \& Technology, vol. 5, pp. 9-11, 2016.

[40] Z. Jiang, J. Li, X. Gao, and N. Fan, "Study on pneumatic wall climbing robot adhesion principle and suction control," in 
Proceedings of the IEEE International Conference on Robotics and Biomimetics, Bangkok Thailand, February 2009.

[41] D. Schmidt and K. Berns, "Climbing robots for maintenance and inspections of vertical structures-a survey of design aspects and technologies," Robotics and Autonomous Systems, vol. 61, no. 12, pp. 1288-1305, 2013.

[42] B. L. Luk, K. P. Liu, A. A. Collie, D. S. Cooke, and S. Chen, "Tele-operated climbing and mobile service robots for remote inspection and maintenance in nuclear industry," Industrial Robot: An International Journal, vol. 33, 2006.

[43] Z.-Y. Qian, Y.-Z. Zhao, Z. Fu, and Q.-X. Cao, International Journal of Advanced Manufacturing Technology, 2006.

[44] K. S. Young, M. L. Chang, I. G. M. Koo, H. Moon, and H. R. Choi, "Development of wall climbing robotic system for inspection purpose," in Proceedings of the IEEE/RSJ International Conference on Intelligent Robots and Systems, Nice, France, September 2008. 